\title{
State Estimation of Linear Systems in the Presence of Sporadic Measurements
}

\author{
Francesco Ferrante $^{\mathrm{a}}$, Frédéric Gouaisbaut ${ }^{\mathrm{a}}$, Ricardo G. Sanfelice ${ }^{\mathrm{b}}$, \\ Sophie Tarbouriech ${ }^{\mathrm{a}}$

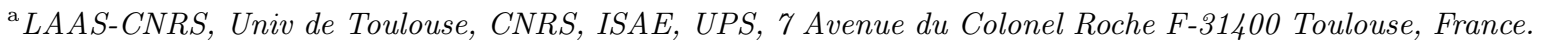 \\ b Univ. of California, Computer Engineering Department, Santa Cruz, CA 95064, USA.
}

\begin{abstract}
This paper deals with the state estimation of linear time-invariant systems for which measurements of the output are available sporadically. To solve the considered problem, we provide an observer with jumps triggered by incoming measurements, which is studied in a hybrid systems framework. Specifically, the resulting system is written in estimation error coordinates and augmented with a timer variable that triggers the event of new measurements arriving. Then, the observer design is performed to achieve global exponential stability (GES) of a closed set including the points for which the state of the plant and its estimate coincide. Furthermore, a computationally tractable design procedure for the proposed observer is presented. Finally, the effectiveness of the proposed methods is demonstrated in two numerical examples.
\end{abstract}

Key words: Hybrid systems, Linear systems, Observers, Convex optimization

\section{Introduction}

Recent technological advances have enabled the control of dynamical systems using data that is transmitted over communication networks. When the networks are not fully reliable, data can get lost or can only be available intermittently $[15,18,31]$. In settings where the controller and the system to control are connected through a network, the classical estimation paradigms of accessing the output of the plant continuously [21] or discretely at desired time instances [5] do not apply and new approaches are required. The limitations of these paradigms have lead to recent efforts in the literature focusing on the impact of intermittent availability of resources in control and estimation. Such works include [32] where state estimation algorithms for networked control systems under the presence of both uncontrolled and controlled sampling are proposed, [23] where a general framework for state estimation for nonlinear networked systems is considered, and [7] where the design, via linear matrix in-

\footnotetext{
* This work has been supported by ANR project LimICoS contract number 12 BS03 00501. Research by R. G. Sanfelice has been partially supported by the National Science Foundation under CAREER Grant no. ECS-1150306 and by the Air Force Office of Scientific Research under YIP Grant no. FA9550-12-1-0366.

Email addresses: francesco.ferrante.2011@ieee.org (Francesco Ferrante), fgouaisb@laas.fr (Frédéric Gouaisbaut), ricardo@ucsc.edu (Ricardo G. Sanfelice), tarbour@laas.fr (Sophie Tarbouriech).
}

equalities, of a state observer-protocol pairs for linear networked systems is presented (in the presence of periodic measurements).

This paper is concerned with the modeling and design of an observer to exponentially estimate the state of a linear time-invariant plant in the presence of sporadically available measurements. As the classical paradigm of continuously measuring the output does not apply to our setting, a suitable observation scheme is needed. To overcome this problem, one may adopt an emulation approach, that is, design an observer while ignoring the lack of continuous information on the measured output, and then replace the measured output by a suitable estimate generated by the most recent measured value. This approach is adopted, for example, in [23]. Another possibility relies on the design of a robust (with respect to bounded sampling time variations) discrete-time observer for the discretized version of the plant. Such an approach can be built upon the results presented, e.g., in [12]. In this paper, we pursue a different approach. Specifically, building from the idea proposed in $[1,24]$ and assuming that the plant input is known, we consider an open-loop observer along with a suitable eventtriggered update law to reset instantaneously the state of the observer. Essentially, the proposed observer has a state that undergoes a jump whenever a new measurement is available. Since the evolution of the proposed observer exhibits both continuous-time behavior and instantaneous changes (not necessarily periodic), we provide a hybrid model capturing the dynamics of the ob- 
server interconnected to the plant. A unique feature of this model is that it is not deterministic; in fact, a setvalued update law is proposed to capture all possible events within a given bounded range. Then, using Lyapunov theory for hybrid systems, we propose a condition that guarantees global exponential stability of a set of points in which the estimation error is zero as well as robustness with respect to bounded perturbations (in an input-to-state stability sense; see [27] and [3]). The proposed approach based on hybrid modeling allows us to effectively exploit the properties of the time domain of the solutions to the resulting hybrid system, in particular, the persistence of jumps. This feature not only provides a better understanding of the system behavior but also enables us to construct a Lyapunov function to certify global exponential stability and characterize the effect of measurement noise via input-to-state stability.

As a second step, our condition guaranteeing global exponential stability is exploited to derive a design algorithm for the proposed observer. To this end, the said condition is first rewritten as a parametric linear matrix inequality. Then, by means of a novel polytopic embedding technique, the obtained parametric linear matrix inequality is turned into a finite number of linear matrix inequalities, whose solution defines suitable parameters for the proposed observer. This result provides a constructive design procedure, which is computationally tractable.

The paper is organized as follows. Section 2 presents the system under consideration, the state estimation problem we solve, and the hybrid modeling of the proposed observer. Section 3 is dedicated to the main results. Section 4 is devoted to numerical issues about the observer synthesis and provides a design algorithm based on linear matrix inequalities for the observer gain. Finally, Section 5 shows the effectiveness of the results presented in two numerical examples.

Notation: The set $\mathbb{N}_{0}$ is the set of the positive integers including zero, $\mathbb{N}$ is the set of the positive integers, and $\mathbb{R}_{\geq 0}$ represents the set of the nonnegative real scalars. For every complex number $\omega, \mathfrak{R e}(\omega)$ and $\mathfrak{I m}(\omega)$ stand respectively for the real and the imaginary part of $\omega$. For a matrix $A \in \mathbb{R}^{n \times m}$, $A^{\prime}$ denotes the transpose of $A,\|A\|$ denotes the induced 2norm, and $\operatorname{He}(A)=A+A^{\prime}$. For two symmetric matrices, $A$ and $B, A>B$ means that $A-B$ is positive definite. In partitioned symmetric matrices, the symbol $\star$ stands for symmetric blocks. For a vector $x \in \mathbb{R}^{n},\|x\|$ denotes the Euclidean norm. Given two vectors $x, y$, we denote $(x, y)=$ $\left[\begin{array}{ll}x^{\prime} & y^{\prime}\end{array}\right]^{\prime}$. Given a set $X$, co $X$ represents the convex hull of $X$. Given a vector $x \in \mathbb{R}^{n}$ and a closed set $\mathcal{A} \subset \mathbb{R}^{n},|x|_{\mathcal{A}}=$ $\inf _{y \in \mathcal{A}}\|x-y\|$. For any function $z: \mathbb{R} \rightarrow \mathbb{R}^{n}$, we denote $z\left(t^{+}\right):=\lim _{s \rightarrow t^{+}} z(s)$. A function $\alpha: \mathbb{R}_{\geq 0} \rightarrow \mathbb{R}_{\geq 0}$ is said to belong to class $\mathcal{K}$ if it is continuous, zero at zero, and strictly increasing. A function $\beta: \mathbb{R}_{\geq 0} \times \mathbb{R}_{\geq 0} \rightarrow \mathbb{R}_{\geq 0}$ is said to belong to class $\mathcal{K} \mathcal{L}$ if it is nondecreasing in its first argument, nonincreasing in its second argument, and $\lim _{s \rightarrow 0^{+}} \beta(s, t)=$ $\lim _{t \rightarrow \infty} \beta(s, t)=0$. Given a function $f: X \rightarrow \mathbb{R}$, ess. sup stands for its essential supremum. For a given real interval
$\mathcal{I}$, and a real matrix $A \in \mathbb{R}^{n \times n}$, we denote $e^{A \mathcal{I}}:=\{Y \in$ $\mathbb{R}^{n \times n}: \exists v \in \mathcal{I}$ such that $\left.Y=e^{A v}\right\}$.

\section{Problem statement}

\subsection{System description}

We consider continuous-time linear time-invariant systems of the form

$$
\begin{aligned}
& \dot{z}=A z+B u \\
& y=M z
\end{aligned}
$$

where $z \in \mathbb{R}^{n}, y \in \mathbb{R}^{q}$, and $u \in \mathbb{R}^{p}$ are, respectively, the state, the measured output, and the input of the system, while $A, B$ and $M$ are constant matrices of appropriate dimensions. We assume that the input $u$ belongs to the class of measurable and locally bounded functions $u:[0, \infty) \rightarrow \mathbb{R}^{p}$. Our goal is to design an observer providing an estimate $\hat{z}$ of the state $z$ with sporadic measurements of $y$; namely, when the output $y$ is available only at some time instances $t_{k}, k \in \mathbb{N}$, not known a priori (a similar setup is considered in [23]). We assume that the sequence $\left\{t_{k}\right\}_{k=1}^{\infty}$ is strictly increasing and unbounded, and that for such a sequence there exist two positive real scalars $T_{1} \leq T_{2}$ such that

$$
\begin{aligned}
0 & \leq t_{1} \leq T_{2} \\
T_{1} & \leq t_{k+1}-t_{k} \leq T_{2} \quad \forall k \in \mathbb{N}
\end{aligned}
$$

As also pointed out in [17], the lower bound $T_{1}$ in condition (2) prevents the existence of accumulation points in the sequence $\left\{t_{k}\right\}_{k=1}^{\infty}$, and, hence, avoids the existence of Zeno behaviors, which are typically undesired in practice. In fact, $T_{1}$ defines a strictly positive minimum time in between two consecutive transmissions. Furthermore, $T_{2}$ defines maximum time in between two consecutive transmissions.

Since the information on the output $y$ is available in an impulsive fashion, motivated by $[1,24]$, to solve the considered estimation problem we design an observer with jumps in its state following the law

$$
\begin{cases}\dot{\hat{z}}(t)=A \hat{z}(t)+B u(t) & \forall t \neq t_{k}, k \in \mathbb{N} \\ \hat{z}\left(t^{+}\right)=\hat{z}(t)+L(y(t)-M \hat{z}(t)) & \forall t=t_{k}, k \in \mathbb{N}\end{cases}
$$

where $L$ is a real matrix of appropriate dimensions to be designed. Note that, in between events, the observer runs in "open-loop" in the sense that no information of the output is used.

Along the lines of [26], the state estimation problem is formulated as a set stabilization problem. Namely, our goal is to design the matrix $L$ such that the set wherein the plant state $z$ and its estimate $\hat{z}$ coincide is globally exponentially stable for the plant (1) interconnected with the observer in (3). At this stage, as usual in estimation problems, we define the estimation error as

$$
\varepsilon:=z-\hat{z} \text {. }
$$


Thus, since at times $t_{k}$ the plant state is unchanged, the error dynamics are given by the following dynamical system with jumps:

$$
\begin{cases}\dot{\varepsilon}(t)=A \varepsilon(t) & \forall t \neq t_{k}, k \in \mathbb{N} \\ \varepsilon\left(t^{+}\right)=(\mathbf{I}-L M) \varepsilon(t) & \forall t=t_{k}, k \in \mathbb{N} .\end{cases}
$$

Due to the linearity of system (1), the estimation error dynamics and the dynamics of $z$ are decoupled. Then, for the purpose of estimation, one can effectively only consider system (5).

\subsection{Hybrid modeling}

The fact that the observer experiences jumps when a new measurement is available and evolves according to a differential equation in between updates suggests that the updating process of the error dynamics can be described via a hybrid system. Due to this, we represent the whole system composed by the plant (1), the observer (3), and the logic triggering jumps as a hybrid system (see [20] where a similar approach is adopted to model a finite-time convergent observer).

To utilize this hybrid systems approach, a model the hidden time-driven mechanism triggering the jumps of the observer is required. To this end, in a similar manner as in [4], we augment the state of the system with an auxiliary timer variable $\tau$ that keeps track of the duration of flows and triggers a jump whenever a certain condition is verified. This additional state allows to describe the time-driven triggering mechanism as a state-driven triggering mechanism, which leads to a model that can be efficiently represented by relying on the framework for hybrid systems proposed in [11]. More precisely, we make $\tau$ to decrease as ordinary time $t$ increases and, whenever $\tau=0$, reset it to any point in $\left[T_{1}, T_{2}\right]$, so as to enforce (2). After each jump, we require the system to flow again. The whole system composed by the estimation error $\varepsilon$ and the timer variable $\tau$ can be represented by the following hybrid system, which we denote $\mathcal{H}_{\varepsilon}$,

$$
\mathcal{H}_{\varepsilon}\left\{\begin{array}{l}
\dot{\varepsilon}=A \varepsilon \\
\dot{\tau}=-1 \\
\varepsilon^{+}=(\mathbf{I}-L M) \varepsilon \\
\tau^{+} \in\left[T_{1}, T_{2}\right]
\end{array}\right\}(\varepsilon, \tau) \in D
$$

where the flow set and the jump set are defined as

$$
\begin{aligned}
& C=\left\{(\varepsilon, \tau) \in \mathbb{R}^{n} \times \mathbb{R}_{\geq 0}: \tau \in\left[0, T_{2}\right]\right\} \\
& D=\left\{(\varepsilon, \tau) \in \mathbb{R}^{n} \times \mathbb{R}_{\geq 0}: \tau=0\right\} .
\end{aligned}
$$

The set-valued jump map captures all possible transmission events within $T_{1}$ or $T_{2}$ units of time. Specifically, the hybrid model in (6) is able to characterize not only the behavior of the analyzed system for a given sequence $\left\{t_{k}\right\}_{k=1}^{\infty}$, but for any sequence satisfying (2). We denote the state of $\mathcal{H}_{\varepsilon}$ by $x=(\varepsilon, \tau)$ and the flow map by $f$ and the jump map by $G$, i.e.,

$$
\begin{array}{ll}
f(x)=\left[\begin{array}{c}
A \varepsilon \\
-1
\end{array}\right] & \forall x \in C \\
G(x)=\left[\begin{array}{c}
(\mathbf{I}-L M) \varepsilon \\
{\left[T_{1}, T_{2}\right]}
\end{array}\right] & \forall x \in D .
\end{array}
$$

Notice that to make the hybrid system (6) an accurate description of the real time-triggered phenomenon, which governs the feedback update process, the variable $\tau$ needs to belong to the interval $\left[0, T_{2}\right]$, property that is guaranteed by the definition of $C$ and $D$.

In this paper, we consider the following notion of global exponential stability ( $G E S$ ) of closed sets for a general hybrid system $\mathcal{H}$ in $\mathbb{R}^{\ell}$; see [28] for more details about this notion.

Definition 1 ([28]) Let $\mathcal{A} \subset \mathbb{R}^{\ell}$ be closed. The set $\mathcal{A}$ is said to be globally exponentially stable (GES) for the hybrid system $\mathcal{H}$ if there exist strictly positive real numbers $\lambda, k$ such that every maximal solution $\phi$ to $\mathcal{H}$ is complete and satisfies for all $(t, j) \in \operatorname{dom} \phi$

$$
|\phi(t, j)|_{\mathcal{A}} \leq k e^{-\lambda(t+j)}|\phi(0,0)|_{\mathcal{A}} .
$$

Then, by introducing the set ${ }^{1}$

$$
\mathcal{A}=\left\{(\varepsilon, \tau) \in \mathbb{R}^{n} \times \mathbb{R}_{\geq 0}: \varepsilon=0, \tau \in\left[0, T_{2}\right]\right\}
$$

the problem to solve is formulated as follows:

Problem 1 Given the matrices $A, B$, and $M$ of appropriate dimensions and two positive scalars $T_{1} \leq T_{2}$, design a matrix $L \in \mathbb{R}^{n \times q}$ such that the set $\mathcal{A}$ defined in (9) is GES for the hybrid system (6).

Remark 1 Concerning existence of solutions to system (6), by relying on the concept of solution proposed in [11, Definition 2.67, it is straightforward to check that for every initial condition $\phi(0,0) \in C \cup D$ there exists at least a nontrivial solution to (6) and that every maximal solution to (6) is complete. Notice that, solving Problem 1 ensures that the estimation error converges exponentially to zero as $t+j$ goes to infinity.

Remark 2 In addition, we can characterize the domain of the solutions to (6). Indeed, the variable $\tau$, acting as a timer, guarantees that for every initial condition $\phi(0,0) \in C \cup D$, the domain of every maximal solution $\phi$ to (6) can be written as follows:

$$
\operatorname{dom} \phi=\bigcup_{j \in \mathbb{N}_{0}}\left(\left[t_{j}, t_{j+1}\right]\right) \times\{j\}
$$

1 By the definition of system (6) and of the set $\mathcal{A}$, for every $x \in C \cup D \cup G(D),|x|_{\mathcal{A}}=\|\varepsilon\|$. 
with $t_{0}=0$ and

$$
\begin{aligned}
T_{1} & \leq t_{j+1}-t_{j} \leq T_{2} \quad \forall j \in \mathbb{N} \\
0 & \leq t_{1} \leq T_{2}
\end{aligned}
$$

where dom $\phi$ is the domain of the solution $\phi$, which is a hybrid time domain; see [11] for more details about solutions to hybrid systems. Furthermore, the structure of the above hybrid time domain implies that for each $(t, j) \in \operatorname{dom} \phi$ we have

$$
t \leq T_{2}(j+1)
$$

the latter relation will play a key role in establishing GES of the set $\mathcal{A}$ for hybrid system (6).

\section{Main results}

\subsection{Conditions for GES}

The following result provides conditions for GES of the set $\mathcal{A}$ defined in (9) for system (6).

Theorem 1 Let $T_{1} \leq T_{2}$ be two given positive real scalars. If there exist a symmetric positive definite matrix $P \in \mathbb{R}^{n \times n}$ and a matrix $L \in \mathbb{R}^{n \times q}$ such that

$$
(\mathbf{I}-L M)^{\prime} e^{A^{\prime} v} P e^{A v}(\mathbf{I}-L M)-P<\mathbf{0} \forall v \in\left[T_{1}, T_{2}\right],
$$

then the set $\mathcal{A}$ defined in (9) is GES for the hybrid system (6).

PROOF. Consider the following Lyapunov function candidate for the hybrid system (6) defined for every $x \in \mathbb{R}^{n} \times \mathbb{R}_{\geq 0}$

$$
V(x)=\varepsilon^{\prime} e^{A^{\prime} \tau} P e^{A \tau} \varepsilon
$$

and notice that there exist two positive scalars $\alpha_{1}, \alpha_{2}$ such that

$$
\alpha_{1}|x|_{\mathcal{A}}^{2} \leq V(x) \leq \alpha_{2}|x|_{\mathcal{A}}^{2} \forall x \in C \cup D \cup G(D) .
$$

Specifically, due to the positive definiteness of $P$ and the nonsingularity of the matrix $e^{A \tau}$ for every $\tau$, by continuity arguments, one can set

$$
\begin{aligned}
& \alpha_{1}=\min _{\tau \in\left[0, T_{2}\right]} \lambda_{\min }\left(e^{A^{\prime} \tau} P e^{A \tau}\right) \\
& \alpha_{2}=\max _{\tau \in\left[0, T_{2}\right]} \lambda_{\max }\left(e^{A^{\prime} \tau} P e^{A \tau}\right)
\end{aligned}
$$

where $\lambda_{\min }(\cdot)$ and $\lambda_{\max }(\cdot)$ denote, respectively, the smallest and the largest eigenvalue of their matrix argument. By straightforward calculations one gets

$$
\nabla V(x)=\left(2 e^{A^{\prime} \tau} P e^{A \tau} \varepsilon, \varepsilon^{\prime} e^{A^{\prime} \tau}\left(A^{\prime} P+P A\right) e^{A \tau} \varepsilon\right) .
$$

Moreover, by exploiting the fact that the matrices $e^{A \tau}$ and $A$ commute, one has

$$
\langle\nabla V(x), f(x)\rangle=0 \quad \forall x \in C .
$$

Now, notice that for every $g \in G(x)$, there exists a real scalar $v$ belonging to the interval $\left[T_{1}, T_{2}\right]$ such that

$$
g=\left[\begin{array}{c}
(\mathbf{I}-L M) \varepsilon \\
v
\end{array}\right] .
$$

Then, for every $g \in G(x)$, one has

$$
\begin{aligned}
V(g)-V(x) & =\varepsilon^{\prime}(\mathbf{I}-L M)^{\prime} e^{A^{\prime} v} P e^{A v}(\mathbf{I}-L M) \varepsilon \\
& -\varepsilon^{\prime} e^{A^{\prime} \tau} P e^{A^{\prime} \tau} \varepsilon .
\end{aligned}
$$

Furthermore, whenever $x \in D$, from (6b), we have that $\tau=0$, which in turn implies

$V(g)-V(x)=\varepsilon^{\prime}\left((\mathbf{I}-L M)^{\prime} e^{A^{\prime} v} P e^{A v}(\mathbf{I}-L M)-P\right) \varepsilon$.

Hence, by virtue of relation (12), it follows that there exists a positive small enough scalar $\beta$ such that, for every $x \in D, g \in G(x)$

$$
V(g)-V(x) \leq-\beta \varepsilon^{\prime} \varepsilon=-\beta|x|_{\mathcal{A}}^{2} .
$$

Without loss of generality, assume that $\alpha_{2}$ in (16) and $\beta$ in (18) satisfy $1-\frac{\beta}{\alpha_{2}}>0$, which is always possible by picking $\beta$ small enough. Define $\theta=\ln \left(1-\frac{\beta}{\alpha_{2}}\right)$ and observe that $\theta<0$. Then

$$
V(g) \leq e^{\theta} V(x) \quad \forall x \in D, g \in G(x) .
$$

Pick

$$
\gamma \in\left(0, \frac{|\theta|}{1+T_{2}}\right] \text { and } R \in\left[\frac{T_{2}|\theta|}{1+T_{2}}, \infty\right) .
$$

Let $\phi$ be a maximal solution to (6). As argued in Remark $1 \phi$ is complete. As shown in the proof of [11, Proposition 3.29], thanks to (17) and (19), direct integration of $(t, j) \mapsto V(\phi(t, j))$ over dom $\phi$ yields

$$
V(\phi(t, j)) \leq e^{\theta j} V(\phi(0,0)) \quad \forall(t, j) \in \operatorname{dom} \phi .
$$

Then, in view of relation (11), by straightforward calculations, it follows that

$$
\theta j \leq R-\gamma(t+j) \quad \forall(t, j) \in \operatorname{dom} \phi
$$

which, along with (14) and (20b), leads to

$$
|\phi(t, j)|_{\mathcal{A}} \leq e^{\frac{R}{2}} \sqrt{\frac{\alpha_{2}}{\alpha_{1}}} e^{-\frac{\gamma}{2}(t+j)}|\phi(0,0)|_{\mathcal{A}} \quad \forall(t, j) \in \operatorname{dom} \phi
$$


hence the set $\mathcal{A}$ defined in (9) is GES for system (6) concluding the proof.

Remark 3 Notice that assuming relation (12) to hold implies that the eigenvalues of $e^{A v}(\mathbf{I}-L M)$ are strictly contained in the unit circle for every $v$ belonging to $\left[T_{1}, T_{2}\right]$. On the other hand, thanks to the Sylvester's determinant theorem, it can be easily proven that for each $v$, the eigenvalues of the matrix $e^{A v}(\mathbf{I}-L M)$ coincide with the eigenvalues of the matrix $(\mathbf{I}-L M) e^{A v}$. Thus, the existence of a pair $P, L$ satisfying condition (12) requires the detectability of the pair $\left(e^{A v}, M e^{A v}\right)$ for each $v$ belonging to $\left[T_{1}, T_{2}\right]$, which in turn, due to the nonsingularity of $e^{A v}$ for any $v$ and for any matrix $A$, is equivalent to the detectability of the pair $\left(e^{A v}, M\right)$. Thus, it follows that Theorem 1 requires the sampled version of system (1) to be detectable for every $v$ belonging to $\left[T_{1}, T_{2}\right]$, though this condition, in general, is only necessary due to $P$ not dependent on $v$. A similar remark is pointed out in [24].

\subsection{Effect of measurement noise}

So far, the measured output was assumed to be perfectly known at sampling times $t_{k}$ with $k \in \mathbb{N}$. However, in a real-world setting, the measured output is affected by measurement noise. To quantify the robustness properties of our observer, denote the measurement noise as $\eta: \mathbb{R}_{>0} \rightarrow \mathbb{R}^{q}$. Then, the measured output is

$$
y=M x+\eta
$$

This, in view of the definition of $\varepsilon$ given in (4), suggests considering the following hybrid system with state $x=$ $(\varepsilon, \tau) \in \mathbb{R}^{n} \times \mathbb{R}_{\geq 0}$ and input $\eta \in \mathbb{R}^{q}$

$$
\mathcal{H}_{\eta}\left\{\begin{array}{l}
\dot{\varepsilon}=A \varepsilon \\
\dot{\tau}=-1
\end{array}\right\}(\varepsilon, \tau) \in C
$$

For notational simplicity, in the sequel we use

$$
\widetilde{G}(x, \eta)=\left[\begin{array}{c}
(\mathbf{I}-L M) \varepsilon-L \eta \\
{\left[T_{1}, T_{2}\right]}
\end{array}\right] .
$$

To study the effect of the measurement noise, we consider the input-to-state-stability (ISS) concept introduced in [27] for continuous-time nonlinear systems and extended to hybrid systems in [3]. For completeness, such a notion is given next for a general hybrid system $\mathcal{H}_{d}$ with state in $\mathbb{R}^{\ell}$ and input $d \in \mathbb{R}^{s}$.

Definition 2 ([3]) A hybrid system $\mathcal{H}_{d}$ is input-tostate-stable with respect to $\mathcal{A}$ if there exist $\gamma \in \mathcal{K} \mathcal{L}$ and $\kappa \in \mathcal{K}$ such that each solution pair $(\phi, d)$ to $\mathcal{H}_{d}$ satisfies

$$
|\phi(t, j)|_{\mathcal{A}} \leq \max \left\{\gamma\left(|\phi(0,0)|_{\mathcal{A}}, t+j\right), \kappa\left(\|d\|_{(t, j)}\right)\right\}
$$

for each ${ }^{2}(t, j) \in \operatorname{dom} \phi$.

Remark 4 Notice that this extension of ISS to hybrid systems deals with hybrid signals as external perturbations. In our case, due to the continuous-time nature of the plant, the perturbation $t \mapsto \eta(t)$ acting on the measured output is a purely continuous-time signal. On the other hand, such a perturbation can be transformed into a hybrid signal, as in [25], to fit in the framework proposed by [3]. In particular, given a continuous time signal $t \mapsto \eta(t)$, in can be easily shown that there exists a solution pair $\left(\phi, \eta_{\mathcal{H}}\right)$ to $(22)$ with

$$
\eta_{\mathcal{H}}(t, j):=\eta(t) \quad \forall(t, j) \in \phi .
$$

Moreover, due to the form of $\eta_{\mathcal{H}}$, the hybrid sup norm $\left\|\eta_{\mathcal{H}}\right\|_{(t, j)}$ in [3] satisfies $\left\|\eta_{\mathcal{H}}\right\|_{(t, j)}=\|\eta\|_{t}$ for every $(t, j) \in \operatorname{dom} \phi$.

Notice that the Lyapunov-like condition for hybrid systems to be ISS given in [3] does not hold for the Lyapunov function in (13) under condition (12) since it does not decrease during flows. Instead, to show ISS of system (22) via the Lyapunov function given in Theorem 1 , we couple strict decrease at jumps of such a function with the persistence of jumps enforced by the variable $\tau$. This claim is formalized in the result given next.

Theorem 2 Let $T_{1} \leq T_{2}$ be two positive real scalars. If there exist a symmetric positive definite matrix $P \in$ $\mathbb{R}^{n \times n}$ and a matrix $L \in \mathbb{R}^{n \times q}$ satisfying condition (12), then the hybrid system (22) is ISS with respect to $\eta$ relatively to the set $\mathcal{A}$.

PROOF. Consider the Lyapunov function defined in (13). Since the measurement noise $\eta$ does not act on the flow map, as in the proof of Theorem 1, (17) holds. For any $(x, \eta) \in \mathbb{R}^{n} \times \mathbb{R}_{\geq 0} \times \mathbb{R}^{q}$ and for each $g \in \widetilde{G}(x, \eta)$ one gets

$$
\begin{aligned}
& V(g)-V(x)=\varepsilon^{\prime}\left((\mathbf{I}-L M)^{\prime} e^{A^{\prime} v} P e^{A v}(\mathbf{I}-L M)\right. \\
& \left.-e^{A^{\prime} \tau} P e^{A \tau}\right) \varepsilon-2 \eta^{\prime} L^{\prime} e^{A^{\prime} v} P e^{A v}(\mathbf{I}-L M) \varepsilon \\
& +\eta^{\prime} L^{\prime} e^{A^{\prime} v} P e^{A v} L \eta
\end{aligned}
$$

2 A pair $(\phi, d)$ is a solution pair to $\mathcal{H}_{d}$ if it satisfies its dynamics; see [3] for more details. Given a hybrid arc $d$, its sup norm at $(t, j) \in \operatorname{dom} d$ is

$$
\left.\begin{array}{c}
\|d\|_{(t, j)}:=\max \left\{\begin{array}{c}
\operatorname{ess.\operatorname {sup}}|d(s, k)| \\
(s, k) \in \operatorname{dom} d \backslash \Gamma(d), s+k \leq t+j
\end{array},\right. \\
\sup _{(s, k) \in \Gamma(d), s+k \leq t+j}|d(s, k)|
\end{array}\right\}
$$

where $\Gamma(d)$ denotes the set of all $(t, j) \in \operatorname{dom} d$ such that $(t, j+1) \in$ dom $d$; see [3] for further details. 
where $v$ is a real scalar belonging to the interval $\left[T_{1}, T_{2}\right]$. Whenever $x \in D$, from (6b), we have $\tau=0$. Then, for each $x \in D, \eta \in \mathbb{R}^{q}, g \in \widetilde{G}(x, \eta)$, one gets

$$
\begin{aligned}
V(g)-V(x) & =\varepsilon^{\prime}\left((\mathbf{I}-L M)^{\prime} e^{A^{\prime} v} P e^{A v}(\mathbf{I}-L M)-P\right) \varepsilon \\
& -2 \eta^{\prime} L^{\prime} e^{A^{\prime} v} P e^{A v}(\mathbf{I}-L M) \varepsilon \\
& +\eta^{\prime} L^{\prime} e^{A^{\prime} v} P e^{A v} L \eta .
\end{aligned}
$$

Moreover, from (12), there exists a small enough positive real scalar $\beta$ such that, for every $v \in\left[T_{1}, T_{2}\right]$ and every $\varepsilon$

$$
\varepsilon^{\prime}\left((\mathbf{I}-L M)^{\prime} e^{A^{\prime} v} P e^{A v}(\mathbf{I}-L M)-P\right) \varepsilon \leq-\beta \varepsilon^{\prime} \varepsilon .
$$

Now recall that for every $a, b \in \mathbb{R}^{n}, 2 a^{\prime} b \leq \omega a^{\prime} a+\omega^{-1} b^{\prime} b$ for every positive real scalar $\omega$. From (26) and (27), setting $a=\varepsilon, b^{\prime}=-\eta^{\prime} L^{\prime} e^{A^{\prime} v} P e^{A v}(\mathbf{I}-L M)$, and $\omega=\frac{\beta}{2}$ yields

$$
\begin{aligned}
& V(g)-V(x) \leq-\frac{1}{2} \beta \varepsilon^{\prime} \varepsilon+\eta^{\prime} \eta \| L^{\prime} e^{A^{\prime} v} P\left(\mathbf{I} \frac{2}{\beta}+\right. \\
&\left.e^{A v}(\mathbf{I}-L M)(\mathbf{I}-L M)^{\prime} e^{A^{\prime} v} P\right) e^{A v} L \|
\end{aligned}
$$

Moreover, thanks to (12), one has $\|(\mathbf{I}-L M)^{\prime} e^{A^{\prime} v} P e^{A v}(\mathbf{I}-$ $L M)\|<\| P \|$. Thus, from (28), it follows $V(g)-V(x) \leq$ $-\frac{1}{2} \beta \varepsilon^{\prime} \varepsilon+\rho\|L\|^{2} \eta^{\prime} \eta$, where

$$
\rho=\|P\|\left(\frac{2}{\beta}+\|P\|\right) \max _{v \in\left[T_{1}, T_{2}\right]}\left(\left\|e^{A^{\prime} v}\right\|^{2}\right) .
$$

The above relationship, together with (14), yields

$$
V(g) \leq e^{\theta} V(x)+\|L\|^{2} \rho \eta^{\prime} \eta \quad \forall x \in D, \eta \in \mathbb{R}^{q}, g \in \widetilde{G}(x, \eta)
$$

where $\theta=\ln \left(1-\frac{\beta}{2 \alpha_{2}}\right)$ and $\alpha_{2}$ is defined in (16). Therefore, from (29) and (17), by considering the domain of the solutions to (22), which is given in (10), it turns out that given any maximal solution pair $(\phi, \eta)$ to $(22)$, one gets

$$
\begin{gathered}
V(\phi(t, 0))=V(\phi(0,0)) \quad \forall t \in\left[0, t_{1}\right] \\
V(\phi(t, j)) \leq e^{\theta j} V(\phi(0,0))+ \\
\rho\|L\|^{2} \sum_{i=0}^{j-1} e^{\theta(j-1-i)}\left\|\eta\left(t_{i+1}, i+1\right)\right\|^{2} \\
\forall(t, j) \in \operatorname{dom} \phi \quad \text { with } j \geq 1
\end{gathered}
$$

Furthermore, with $\theta$ negative as in the proof of Theorem 1 , for each $(t, j) \in \operatorname{dom} \phi$ such that $j \geq 1$, we have

$$
V(\phi(t, j)) \leq e^{\theta j} V(\phi(0,0))+\frac{\rho e^{-\theta}\|L\|^{2}}{e^{-\theta}-1}\|\eta\|_{(t, j)}^{2} .
$$

Since the input dependent term in the right-hand side of (31) is nonnegative, by combining it with (30a) and $(31)$, we obtain, for each $(t, j) \in \operatorname{dom} \phi$,

$$
V(\phi(t, j)) \leq e^{\theta j} V(\phi(0,0))+\frac{\rho e^{-\theta}\|L\|^{2}}{e^{-\theta}-1}\|\eta\|_{(t, j)}^{2},
$$

further using (14) one gets

$$
|\phi(t, j)|_{\mathcal{A}}^{2} \leq \frac{\alpha_{2}}{\alpha_{1}} e^{\theta j}|\phi(0,0)|_{\mathcal{A}}^{2}+\frac{\rho e^{-\theta}\|L\|^{2}}{\left(e^{-\theta}-1\right) \alpha_{1}}\|\eta\|_{(t, j)}^{2}
$$

Now, by following the same arguments in the proof of Theorem 1, for some (solution independent) positive real scalars $\gamma, R$, from (33) one gets

$$
|\phi(t, j)|_{\mathcal{A}}^{2} \leq e^{-\gamma(t+j)} e^{R} \frac{\alpha_{2}}{\alpha_{1}}|\phi(0,0)|_{\mathcal{A}}^{2}+\frac{\rho e^{-\theta}\|L\|^{2}}{\left(e^{-\theta}-1\right) \alpha_{1}}\|\eta\|_{(t, j)}^{2}
$$

or equivalently

$$
\begin{array}{r}
|\phi(t, j)|_{\mathcal{A}} \leq \max \left\{\sqrt{2 \frac{\alpha_{2}}{\alpha_{1}} e^{\frac{R}{2}} e^{-\frac{\gamma(t+j)}{2}}|\phi(0,0)|_{\mathcal{A}},}\right. \\
\left.\sqrt{\frac{2 \rho e^{-\theta}}{\left(e^{-\theta}-1\right) \alpha_{1}}}\|L\|\|\eta\|_{(t, j)}\right\}
\end{array}
$$

Thus, according to Definition 2, the hybrid system (22) is ISS with respect to $\eta$ (relatively to the set $\mathcal{A}$ ).

Remark 5 The above result allows to conclude that condition (12) actually suffices to guarantee the ISS property for hybrid system (22). Essentially, in the considered case, we do not need to find an ISS Lyapunov function as defined in [3] to prove input-to-state stability. In fact, in the presence of persistent jumps, the availability of a weak Lyapunov function like the one we employ is typically enough.

Remark 6 We would like to point out that other approaches could be used to perform stability and ISS analysis of the estimation error dynamics (5); see, e.g., [16, 30]. However, our approach has some notable advantages. The first advantage is that, thanks to the choice of the Lyapunov function $V$ we considered in Theorem 1 and Theorem 2, the intersample behavior is directly accounted by a single application of Lyapunov theory for hybrid systems. This allows to derive in one step an explicit (hybrid) exponential bound of the estimation error that holds not only at times $t_{k}$ but at any time $t$. The second advantage is that, due to the fact that our constructions lead to a well-posed hybrid system (as defined in [11]), our approach allows to argue that the convergence properties of the estimation error are robust to general small perturbations; see [11]. 


\section{Numerical Design Procedure}

In the previous section a condition to guarantee global exponential stability and input-to-state-stability, respectively, for systems (6) and (22) was provided. However, due to its form, such a condition is not computationally tractable to obtain a solution to Problem 1. Indeed, from a numerical standpoint, condition (12) has two drawbacks: it is nonlinear in $P$ and $L$, and it needs to be verified for infinitely many values of $v$. The relevance of the second drawback is evident at a first sight, while the lack of lineariry is a severe constraint, since the solution to nonlinear matrix inequalities problems often lead to NP-hard problems; see e.g., [2]. Thus, in order to make the problem numerically tractable, further work is needed. To this end, the following result provides a first step toward a design procedure for the proposed observer based on the solution to linear matrix inequalities.

Proposition 1 Let $T_{1} \leq T_{2}$ be two given positive real scalars. The matrices $P$ and $L$ satisfy condition (12), if and only if there exists a matrix $F \in \mathbb{R}^{n \times n}$ such that for every $v \in\left[T_{1}, T_{2}\right]$

$$
\left[\begin{array}{ccc}
-\operatorname{He}(F) & F-F L M & e^{A^{\prime} v} P \\
\star & -P & \mathbf{0} \\
\star & \star & -P
\end{array}\right]<\mathbf{0}
$$

PROOF. Set

$$
Z=\left[\begin{array}{cc}
e^{A v} P e^{A^{\prime} v} & \mathbf{0} \\
\mathbf{0} & -P
\end{array}\right], S=\left[\begin{array}{c}
(\mathbf{I}-L M) \\
\mathbf{I}
\end{array}\right], Y=\left[\begin{array}{l}
\mathbf{0} \\
\mathbf{I}
\end{array}\right]
$$

Then, condition (12) can be rewritten as

$$
S^{\prime} Z S<\mathbf{0}
$$

while the positive definiteness of $P$ can be expressed equivalently by requiring that

$$
Y^{\prime} Z Y<\mathbf{0}
$$

Thus, by the projection lemma [22], (37) and (38) are satisfied if and only if there exists a matrix $F$ such that

$$
\left[\begin{array}{cc}
e^{A^{\prime} v} P e^{A v}-\operatorname{He}(F) & F-F L M \\
\star & -P
\end{array}\right]<\mathbf{0} .
$$

Moreover, by Schur complement, from (39) one gets

$$
\left[\begin{array}{ccc}
-\operatorname{He}(F) & F-F L M & e^{A^{\prime} v} \\
\star & -P & \mathbf{0} \\
\star & \star & -P^{-1}
\end{array}\right]<\mathbf{0}
$$

and finally, pre-and-post multiplying by $\operatorname{diag}\{\mathbf{I}, \mathbf{I}, P\}$ yields the left-hand side matrix in (36), concluding the proof.

Remark 7 The above result avoids the conservatism introduced by Proposition 1 in [8] by showing that condition (36) is actually equivalent to (12).

Remark 8 Notice that by setting $F L=J$, condition (36) turns into a parametric LMI in $v$, with respect to the unknown matrices $F, J$, and $P$.

Proposition 1, along with the Remark 7, provides an equivalent condition to (12), which is linear in the decision variable $F, J$ and $P$. Nevertheless, the obtained condition still has to be verified for infinitely many values of $v$. This situation is rather common in the literature of sampled-data and impulsive systems; see, e.g.,[17] and the references therein. A general procedure to overcome this issue consists in embedding the term $e^{A v}$, with $v$ in the interval $\left[T_{1}, T_{2}\right]$, into a polytope, (a convex set having a finite number of extreme points). Namely, one needs to find some matrices $X_{1}, X_{2}, \ldots, X_{\nu} \in \mathbb{R}^{n \times n}$ such that $e^{A v} \in \operatorname{co}\left\{X_{1}, X_{2}, \ldots, X_{\nu}\right\}$ whenever $v \in\left[T_{1}, T_{2}\right]$. Throughout the sequel, we refer to such a polytope as polytopic overapproximation or polytopic embedding of $e^{A v}$ on $\left[T_{1}, T_{2}\right]$. Then, by exploiting the convexity of condition (36), one can obtain a finite set of inequalities, whose satisfaction implies (36) to hold. This proposed approach is formalized for our case in the result given next.

Corollary 1 Let $T_{1} \leq T_{2}$ be two given positive scalar and let $\left\{X_{1}, X_{2}, \ldots, X_{\nu}\right\}$ be matrices such that $e^{A\left[T_{1}, T_{2}\right]} \in \operatorname{co}\left\{X_{1}, X_{2}, \ldots, X_{\nu}\right\}$. If there exist a symmetric positive definite matrix $P \in \mathbb{R}^{n \times n}$, a matrix $J \in \mathbb{R}^{n \times q}$, and a matrix $F \in \mathbb{R}^{n \times n}$ such that, for every $i \in\{1, \ldots, \nu\}$,

$$
\left[\begin{array}{ccc}
-\operatorname{He}(F) & F-J M & X_{i}^{\prime} P \\
\star & -P & \mathbf{0} \\
\star & \star & -P
\end{array}\right]<\mathbf{0}
$$

then the matrices $P$ and $L=F^{-1} J$ satisfy condition (12).

PROOF. The proof directly follows from the linearity of (36) with respect to $e^{A v}$.

\subsection{Polytopic embedding}

The derivation of a polytopic overapproximation of the exponential matrix on a given compact interval is recognized in the literature as a difficult problem; see $[6,14]$. In [14] an exhaustive comparison between several kinds of overapproximations is presented and the authors suggest that two classes of approaches can be pursued to determine polytopic overapproximations of 
the matrix exponential term on a given compact interval. The first approach aims at determining a finite number of matrices $F_{1}, F_{2}, \ldots, F_{\nu} \in \mathbb{R}^{n \times n}$ such that $e^{A \mathcal{I}} \in \operatorname{co}\left\{F_{1}, F_{2}, \ldots, F_{\nu}\right\}$ for a given compact interval $\mathcal{I}$. This approach is commonly called without uncertainties. The other approach leads to a finite number of matrices $F_{1}, F_{2}, \ldots, F_{\mu} \in \mathbb{R}^{n \times n}$ and a norm bounded uncertainty $\Delta(v) \in \mathbb{R}^{n \times n}$ such that, for every $v$ belonging to a given compact interval, $e^{A v}=\sum_{i=1}^{\mu} \alpha_{i}(v) F_{i}+\Delta(v)$ for some positive scalar functions $\alpha_{1}, \ldots, \alpha_{\mu}$ with $\sum_{i=1}^{\mu} \alpha_{i}(v)=$ 1. This approach is commonly called with uncertainties. On the one hand, the approaches with uncertainties, in general, obtains tighter overapproximations than those without uncertainties; see [6]. On the other hand, managing bounded uncertainties to build a design procedure can be hard, although in [16] a possible two-stage design procedure is proposed to cope with this issue.

In this paper, we propose a novel methodology to build a polytopic embedding without uncertainties. Such a methodology is based on the well known expansion of the matrix exponential based on residue matrices. In particular, by arranging the eigenvalues of the matrix $A$ in a way such that the first $\sigma_{r}$ are real and distinct, the following $\sigma_{c}$ are complex and distinct, and the remaining $\sigma_{c}$ are the conjugates of the previous ones, such an expression is given by

$$
\begin{aligned}
e^{A v} & =\sum_{i=1}^{\sigma_{r}} \sum_{j=1}^{m_{i}^{r}} R_{i j} e^{\lambda_{i} v} \frac{v^{j-1}}{(j-1) !}+ \\
& +\sum_{i=1}^{\sigma_{c}} \sum_{j=1}^{m_{i}^{c}} 2 e^{\mathfrak{R e}\left(\lambda_{i}\right) v}\left(\mathfrak{R e}\left(R_{i j}\right) \cos \left(\mathfrak{I m}\left(\lambda_{i}\right) v\right)\right. \\
& \left.-\mathfrak{I m}\left(R_{i j}\right) \sin \left(\mathfrak{I m}\left(\lambda_{i}\right) v\right)\right) \frac{v^{j-1}}{(j-1) !}
\end{aligned}
$$

where $\sigma_{r}$ is the number of distinct real eigenvalues, and $\sigma_{c}$ the number of distinct complex-conjugate eigenvalue pairs. The constants $m_{i}^{r}$ and $m_{i}^{c}$ are, respectively, the multiplicity of the real eigenvalue $\lambda_{i}$ and of the complexconjugate eigenvalue pair $\lambda_{i}, \lambda_{i}^{*}$ in the minimal polynomial of the matrix $A$. The matrices $R_{i j}$ are real $n \times n$ matrices corresponding to the residues associated to the partial fraction expansion of $(s I-A)^{-1}$. The advantage of the proposed method lies in the fact that there exist several methods to compute the residues matrices. For instance, in this work, we rely on the procedure proposed in [19].

Once the residue matrices are known, to build a polytopic embedding of $e^{A v}$ on the considered interval, one can proceed in a similar manner as in [6]. Namely, by defining the following matrices

$$
\begin{aligned}
& \left\{X_{1}, X_{2}, \ldots, X_{\nu}\right\}=\left\{\sum_{i=1}^{\sigma_{r}} \sum_{j=1}^{m_{i}^{r}} R_{i j} \beta_{i j}\right. \\
& +\sum_{i=1}^{\sigma_{c}} \sum_{j=1}^{m_{i}^{c}} \gamma_{i j} \mathfrak{R e}\left(R_{i j}\right)+\gamma_{i j}^{*} \mathfrak{I m}\left(R_{i j}\right): \beta_{i j} \in\left\{\overline{\beta_{i j}}, \underline{\beta_{i j}}\right\}, \\
& \left.\gamma_{i j} \in\left\{\overline{\gamma_{i j}}, \underline{\gamma_{i j}}\right\}, \gamma_{i j}^{*} \in\left\{\overline{\gamma_{i j}^{*}}, \underline{\gamma_{i j}^{*}}\right\}\right\} 8
\end{aligned}
$$

where

$$
\begin{aligned}
& \overline{\beta_{i j}}=\max _{v \in\left[T_{1}, T_{2}\right]} e^{\lambda_{i} v} \frac{v^{j-1}}{(j-1) !} \\
& \underline{\beta_{i j}}=\min _{v \in\left[T_{1}, T_{2}\right]} e^{\lambda_{i} v} \frac{v^{j-1}}{(j-1) !} \\
& \overline{\gamma_{i j}}=\max _{v \in\left[T_{1}, T_{2}\right]} 2 e^{\Re \mathfrak{R}\left(\lambda_{i}\right) v} \cos \left(\mathfrak{I m}\left(\lambda_{i}\right) v\right) \frac{v^{j-1}}{(j-1) !} \\
& \underline{\gamma_{i j}}=\min _{v \in\left[T_{1}, T_{2}\right]} 2 e^{\Re \mathfrak{R}\left(\lambda_{i}\right) v} \cos \left(\mathfrak{I m}\left(\lambda_{i}\right) v\right) \frac{v^{j-1}}{(j-1) !} \\
& \overline{\gamma_{i j}^{*}}=\max _{v \in\left[T_{1}, T_{2}\right]}-2 e^{\mathfrak{R e}\left(\lambda_{i}\right) v} \sin \left(\mathfrak{I m}\left(\lambda_{i}\right) v\right) \frac{v^{j-1}}{(j-1) !} \\
& \underline{\gamma_{i j}^{*}}=\min _{v \in\left[T_{1}, T_{2}\right]}-2 e^{\Re \mathfrak{R}\left(\lambda_{i}\right) v} \sin \left(\mathfrak{I m}\left(\lambda_{i}\right) v\right) \frac{v^{j-1}}{(j-1) !} .
\end{aligned}
$$

It is straightforward to show that for each $v \in\left[T_{1}, T_{2}\right]$, $e^{A v} \in \operatorname{co}\left\{X_{1}, X_{2}, \ldots, X_{\nu}\right\}$. Furthermore, the number of elements generating the latter convex-hull is always finite, in particular $\nu \leq 2^{n}$.

Remark 9 The most laborious part of the proposed technique, namely the computation of the residue matrices, does not depend on the considered interval $\left[T_{1}, T_{2}\right]$. Thus, for a given matrix $A$, once the residues are known and stored, the construction of the needed polytopic embedding only requires the computation of the extrema of a finite number of continuous scalars functions on a compact interval. Notice that although the proposed embedding technique could lead to similar results to the ones proposed in [6], our methodology does not require either the derivation of the real Jordan form of $A$ or its minimal polynomial. Moreover, the proposed methodology is systematic and does not require dedicated strategies depending on the multiplicity of the eigenvalues.

Remark 10 A different approach could be taken to solve the observer design problem considered in this paper. Indeed, based on a discretization of system (1) over the interval $\left[t_{k}, t_{k+1}\right]$, the uncertainty on the sampling times could be embedded in a linear parameter varying (LPV) system framework. Then, the bounds of the uncertain parameter of the resulting LPV system would be directly related to the bounds $T_{1}$ and $T_{2}$ in (2). From this, an $L P V$ observer could be designed by using the results in [12, 13, 29]. In this paper, we have chosen an alternative way to solve the observer design problem by relying on the hybrid dynamical systems framework in [11]. Pursuing this approach allows to precisely and directly characterize the intersample behavior of the error dynamics via Lyapunov analysis, as well as to guarantee that state estimation is (practically) preserved in the presence of small 
perturbations. Such perturbations can be chosen to be very general allowing to encompass several situations of practical interest, as for instance the case of small variations of the sampling period bounds $T_{1}$ and $T_{2}$; see [11] for further details on perturbed hybrid systems. Somehow, LPV and hybrid approaches can be viewed as complementary not only in terms of modeling but also in terms of the way to design the observer gain. For example, the design conditions proposed in [12] lead to a parameter dependent gain, whereas the gain obtained via our approach is independent of the sampling bounds. Moreover, the polytopic embedding used in the computational procedure to solve our conditions is different from the convex combination structure proposed in [12].

\section{Numerical Examples}

Example 1 In this first example, we want to further highlight the capabilities offered by the proposed methodology. Let us consider the scalar system defined by the following data $A=1, M=1$, and assume that $T_{2}=T_{1}=1$. By taking $P=1$, which in turns yields $\alpha_{1}=1, \alpha_{2}=e^{2}$, it turns out that (12) can be verified by ensuring that $|1-L|<e^{-1}$. In particular, by picking $L=1.1-e^{-1} \approx$ 0.7321 , one gets $\beta=\left|(1-L M)^{2} e^{2}-1\right| \approx 0.4698, \theta=$ $\ln \left(1-\frac{\beta}{\alpha_{2}}\right) \approx-0.0657, R=\gamma \approx 0.0328$. Fig. 1 shows the evolution of the norm of the estimation error along with the exponential bound obtained via Theorem 1. As argued, the exponential bound we provide jumps along the solution.

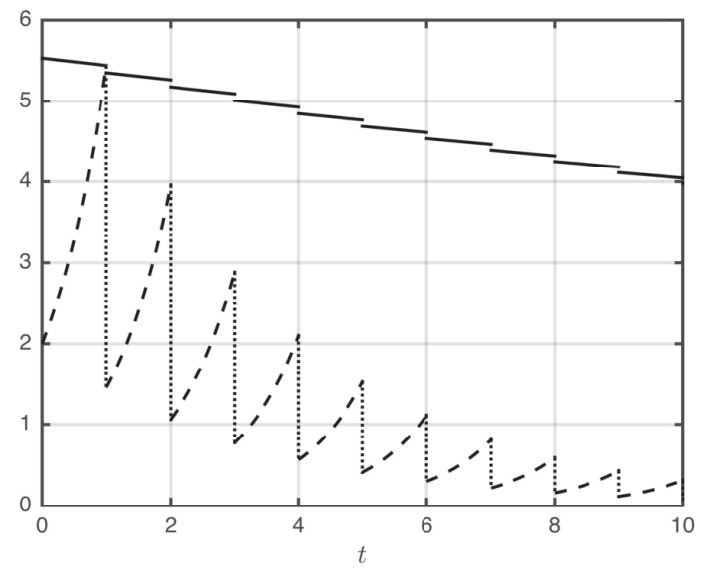

Fig. 1. The evolution of the estimation error (dashed) and its exponential bound (solid) both projected onto ordinary time. Vertical dotted lines stand for instantaneous jumps in the estimation error trajectory.

Example 2 Consider the mass-spring system in [10], defined as follows

$$
\dot{z}=\underbrace{\left[\begin{array}{cccc}
0 & 0 & 1 & 0 \\
0 & 0 & 0 & 1 \\
-2 & 1 & -1 & 0 \\
2 & -2 & 0 & -2
\end{array}\right]}_{A} z+\underbrace{\left[\begin{array}{l}
0 \\
0 \\
1 \\
0
\end{array}\right]}_{B} u
$$

where $z_{1}, z_{2}$ are respectively the position of the first and the second mass, while $z_{3}$ and $z_{4}$ are respectively the speed of the first and the second mass, and $u$ is the force applied to the second mass. Suppose that only $z_{1}$ is measurable through a biased sensor which can be accessed at most every $0.2 s$ and at least every $3 s$. That is, assuming the initial time $t_{0}=0$, the measured output can be expressed as

$$
y\left(t_{k}\right)=z_{1}\left(t_{k}\right)+b \quad \forall k \in \mathbb{N}
$$

where $\left\{t_{k}\right\}_{k=1}^{\infty}$ is an increasing and unbounded sequence of positive times satisfying (2) with $T_{1}=0.2$, and $T_{2}=3$, and $b$ is the sensor bias, i.e., an unknown real constant.

We want to build an observer providing an exponential estimate of the state $z$. To fit this problem in the setting addressed by Theorem 1, one needs to avoid considering the bias as an external perturbation. To this end, we follow an exosystem approach; see [9]. Namely, we model the constant bias affecting the output sensor as an extra state, $b$, such that $\dot{b}=0$. In this way, $y=M \bar{z}$, where $M:=\left[\begin{array}{lllll}1 & 0 & 0 & 0 & 1\end{array}\right]$ and $\bar{z}:=(z, b)$. Therefore, by taking $\bar{z}$ as vector state, one can consider the extended system defined by $\bar{A}=\left[\begin{array}{ll}A & \mathbf{0} \\ \mathbf{0} & \mathbf{0}\end{array}\right], \bar{B}^{\prime}=\left[\begin{array}{ll}B^{\prime} & 0\end{array}\right]$ that matches the class of systems considered in this paper. By building a polytopic embedding following the technique proposed in Section 4 , with $T_{1}=0.2$ and $T_{2}=3$, via Corollary 1 , one gets

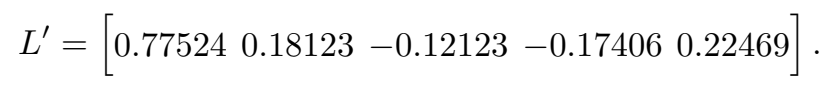

Assuming $u(t)=\sin (t), b=1$, and denoting the estimate provided by the observer as $\hat{z}_{e}:=(\hat{z}, \hat{b})$. Figure 2 reports the evolution of the squared norm of estimation error projected both onto ordinary time and onto jump time. Simulations show that the estimation error converges to zero as $t+j$ goes to infinity.

\section{Conclusion}

This paper proposed a methodology to model and design, through the solution to some linear matrix inequalities, a measurement-triggered observer to exponentially estimate the state of a linear plant in the presence of sporadically available measurements. The considered observer is shown to be ISS with respect to measurement noise. Moreover, a design procedure based on the solution to linear matrix inequalities, along with a novel polytopic embedding technique were proposed. Furthermore, the effectiveness of the proposed methodology is displayed in a numerical example. In particular, this paper shows that the hybrid systems framework proposed in [11] efficiently models and analyzes the estimation error dynamics to prove exponential state estimation via Lyapunov arguments.

Future research directions include the design of an observer-based controller, whose core is centered on the proposed observer, as well as robustness analysis in the presence of large uncertainties on the plant model data. 

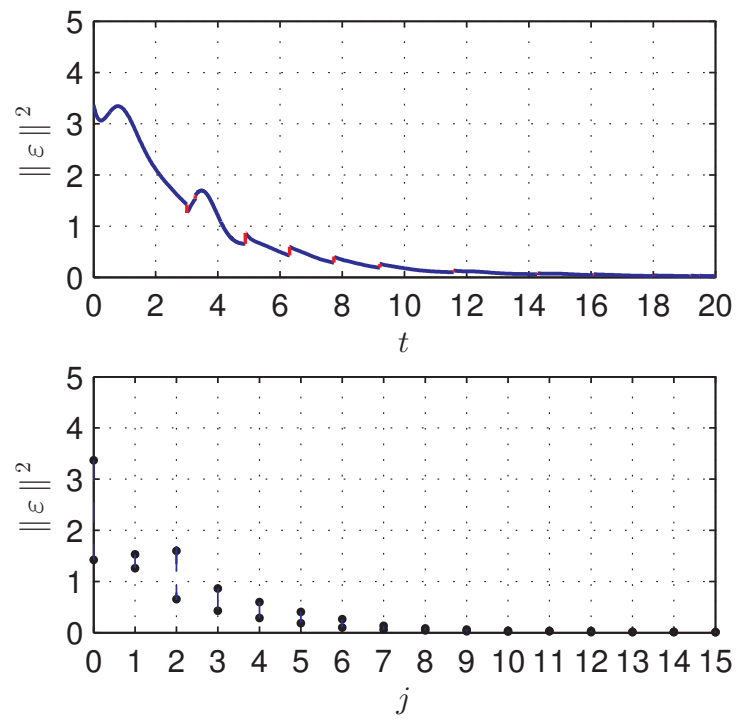

Fig. 2. The evolution of $\|\varepsilon\|^{2}$ projected onto ordinary time $t$ and onto jump time $j$.

Another interesting future outlook concerns the evaluation of the performances, in terms of convergence speed, offered by the proposed observer compared with the observer schemes derived via emulation approach as those analyzed in [23]. Indeed, the main peculiarity of the proposed scheme is that at every jump the whole state of the observer is reset. These instantaneous changes in the observer dynamics can potentially lead to an improvement of the convergence rate, while avoiding the need of a large observer gain.

\section{References}

[1] V. Andrieu, M. Nadri, U. Serres, and J. Vivalda. Continuous discrete observer with updated sampling period. In NOLCOS 2013, volume 9, pages 439-444, 2013.

[2] S. Boyd, L. E. Ghaoui, E. Feron, and V. Balakrishnan. Linear Matrix Inequalities in System and Control Theory. Society for Industrial and Applied Mathematics, June 1997.

[3] C. Cai and A. R. Teel. Characterizations of inputto-state stability for hybrid systems. Systems $\&$ Control Letters, 58(1):47-53, 2009.

[4] D. Carnevale and A. R. Teel. A Lyapunov proof of an improved maximum allowable transfer interval for networked control systems. IEEE Transactions on Automatic Control, 52(5):892, 2007.

[5] T. Chen, B. Francis, and T. Hagiwara. Optimal sampled-data control systems. Proceedings of the IEEE, 86(4):741-741, 1998.

[6] M. B. G. Cloosterman, L. Hetel, N. van de Wouw, W. P. M. H. Heemels, J. Daafouz, and H. Nijmeijer. Controller synthesis for networked control systems. Automatica, 46(10):1584-1594, 2010.

[7] D. Dačić and D. Nešić. Observer design for wired linear networked control systems using matrix inequalities. Automatica, 44(11):2840-2848, 2008.
[8] F. Ferrante, F. Gouaisbaut, R. G. Sanfelice, and S. Tarbouriech. An observer with measurementtriggered jumps for linear systems with known input. In Proceedings of the 19th World Congress of the International Federation of Automatic Control, volume 19, pages 140-145, 2014. Available online at https://hybrid.soe.ucsc.edu/biblio.

[9] B. A. Francis and W. M. Wonham. The internal model principle of control theory. Automatica, 12(5):457-465, 1976.

[10] J. C. Geromel and M. C. de Oliveira. $H_{2}$ and $H_{\infty}$ robust filtering for convex bounded uncertain systems. IEEE Transactions on Automatic Control, 46(1):100-107, 2001.

[11] R. Goebel, R. G. Sanfelice, and A. R. Teel. Hybrid Dynamical Systems: Modeling, Stability, and Robustness. Princeton University Press, 2012.

[12] M. Halimi, G. Millerioux, and J. Daafouz. Polytopic observers for lpv discrete-time systems. In Robust Control and Linear Parameter Varying Approaches, pages 97-124. Springer, 2013.

[13] W. P. M. H. Heemels, J. Daafouz, and G. Millerioux. Observer-based control of discrete-time lpv systems with uncertain parameters. IEEE Transactions on Automatic Control, 55(9):2130-2135, 2010.

[14] W. P. M. H. Heemels, N. van de Wouw, R. H. Gielen, M. C. F. Donkers, L. Hetel, S. Olaru, M. Lazar, J. Daafouz, and S. Niculescu. Comparison of overapproximation methods for stability analysis of networked control systems. In Proceedings of the 13th ACM International Conference on Hybrid Systems: Computation and Control, pages 181-190. ACM, 2010.

[15] J. P. Hespanha, P. Naghshtabrizi, and Y. Xu. A survey of recent results in networked control systems. Proceeding of the IEEE, 95(1):138, 2007.

[16] L. Hetel, J. Daafouz, and C. Iung. Stabilization of arbitrary switched linear systems with unknown time-varying delays. IEEE Transactions on Automatic Control, ,51(10):1668-1674, Oct 2006.

[17] L. Hetel, J. Daafouz, S. Tarbouriech, and C. Prieur. Stabilization of linear impulsive systems through a nearly-periodic reset. Nonlinear Analysis: Hybrid Systems, 7(1):4-15, 2013.

[18] D. Hristu-Varsakelis and W. S. Levine. Handbook of networked and embedded control systems. Springer, 2005.

[19] J. Leyva-Ramos. A new look at partial fraction expansion of transfer function matrices from a computational viewpoint. Computers \& Mathematics with Applications, 26(3):27-35, 1993.

[20] Y. Li and R. G. Sanfelice. A robust finite-time convergent hybrid observer for linear systems. In Proceedings of the 52th IEEE Conference on Decision and Control, 2013.

[21] D. G. Luenberger. An introduction to observers. IEEE Transactions on Automatic Control, 16(6), 1971. 
[22] G. Pipeleers, B. Demeulenaere, J. Swevers, and L. Vandenberghe. Extended LMI characterizations for stability and performance of linear systems. Systems \& Control Letters, 58(7):510-518, 2009.

[23] R. Postoyan and D. Nešić. A framework for the observer design for networked control systems. IEEE Transactions on Automatic Control, 57(5):13091314, 2012.

[24] T. Raff and F. Allgöwer. Observers with impulsive dynamical behavior for linear and nonlinear continuous-time systems. In Proceedings of the 46th IEEE Conference on Decision and Control, pages 4287-4292, 2007.

[25] M. Robles and R. G. Sanfelice. Hybrid controllers for tracking of impulsive reference state trajectories: a hybrid exosystem approach. In Proceedings of the 14th international conference on Hybrid systems: Computation and Control. ACM, 2011.

[26] R. G. Sanfelice and L. Praly. Convergence of nonlinear observers on $\mathbb{R}^{n}$ with a Riemannian metric (part I). IEEE Transactions on Automatic Control, 57(7):1709, 2012.

[27] E. D. Sontag. Smooth stabilization implies coprime factorization. IEEE Transactions on Automatic
Control, 34(4):435-443, 1989.

[28] A. R. Teel, F. Forni, and L. Zaccarian. Lyapunovbased sufficient conditions for exponential stability in hybrid systems. IEEE Transactions on Automatic Control, 2013.

[29] R. Tóth. Modeling and identification of linear parameter-varying systems, volume 403. Springer, 2010.

[30] N. van de Wouw, P. Naghshtabrizi, M.B.G. Cloosterman, and J. P. Hespanha. Tracking control for sampled-data systems with uncertain time-varying sampling intervals and delays. International Journal of Robust and Nonlinear Control, 20(4):387-411, 2010.

[31] G. C. Walsh, Hong Y., and L. G. Bushnell. Stability analysis of networked control systems. IEEE Transactions on Control Systems Technology, 10(3):438446, May 2002.

[32] Y. Xu and J. P. Hespanha. Estimation under uncontrolled and controlled communications in networked control systems. In Proceedings of the 44th IEEE Conference on Decision and Control and European Control Conference 2005, pages 842-847, 2005 . 